\title{
The use of prescribed opioid analgesics \& the risk of serious infections
}

\author{
Andrew D Wiese ${ }^{*, 1}$ \& Carlos G Grijalva ${ }^{1,2}$ \\ ${ }^{1}$ Department of Health Policy, Vanderbilt University Medical Center, $150021^{\text {st }}$ Avenue South, Nashville, TN, 37212, USA \\ ${ }^{2}$ Mid-South Geriatric Research Education \& Clinical Center, VA Tennessee Valley Health Care System, $131024^{\text {th }}$ Avenue S, \\ Nashville, TN, 37212, USA \\ *Author for correspondence: Tel.: +1 615875 7997; andrew.d.wiese.1@vumc.org

\section{“current evidence strongly suggests that opioid analgesic use is a risk factor for serious infections"}

First draft submitted: 30 March 2018; Accepted for publication: 20 April 2018; Published online: 13 June 2018

Keywords: immunosuppression • opioid analgesics • serious infections

The opioid epidemic in the USA was recently declared a public health emergency [1]. Opioid analgesics are a class of medications used primarily for the treatment of pain [1]. The substantial increase in opioid analgesic use during the past several years has been well documented. There were approximately 92 million US adults using prescription opioids in 2015, about three-times more users than in 1999 [2]. Although some data suggest that the increasing use of opioid analgesics has slowed down in more recent years, opioid analgesic use remains substantial and has been continually increasing in some geographical regions of the USA [2]. There are similar concerns regarding increasing opioid analgesic use in other countries as well [3]. Unfortunately, despite the high prevalence of opioid use, our understanding of the long-term safety and effectiveness of opioid analgesics remains incomplete [4].

Well-recognized, common side effects of opioid analgesic use include sedation, nausea, vomiting and constipation. Less common side effects include hyperalgesia, muscle rigidity and hormonal imbalances [5]. Unfortunately, the small sample sizes and short duration of existing randomized controlled trials limit the ability to detect more serious but less frequent adverse outcomes linked to widespread and long-term opioid use. However, recent epidemiologic evidence suggests that prescription opioid analgesic use is associated with an increased risk of opioid use disorders, overdoses, cardiovascular events and adverse respiratory outcomes that contribute to the increased morbidity and mortality reported among opioid users compared with nonusers [4,6-10]. Still, these specific outcomes do not fully account for the excess morbidity and mortality observed among opioid users. In fact, a growing body of evidence indicates that an increased risk of serious infections represents another clinically important adverse outcome associated with opioid use [11,12].

\section{Experimental evidence of opioid-induced immunosuppression \& susceptibility to infections}

Results from experimental and in vitro studies have demonstrated that certain opioids can negatively impact the immune response through the downregulation of innate and acquired immune pathways [11,12]. Morphine, the prototypical opioid and an active metabolite of some other opioids, has an affinity for binding to the mu opiate (MOP) receptor found on T lymphocytes, macrophages and immature immune cells [11,12]. Morphine exposure is also linked to the disruption of natural killer cell activity, as well as the disruption of macrophage and lymphocyte production, migration and phagocytosis [11,12]. Morphine also impacts macrophage activity through interaction with IL-2 and IFN- $\gamma$, and through the induction of macrophage apoptosis [11,12]. Morphine exposure is also thought to impair the immune response further by downregulating major histocompatibility complex (MHC)class II receptors on B cells (thereby reducing T-cell activation) and by binding to T-cell MOP receptors and impacting specific T-cell phenotype activation [11,12].

Although most studies of opioid-induced immunosuppression focused on morphine, the immunosuppressive effects of opioids are not limited to morphine. Both codeine and morphine reduce natural killer cell activity and

Future Medicine 
IL-2 production compared with hydromorphone or oxycodone in animal models [13]. Similarly, fentanyl inhibits lymphocyte and natural killer cell proliferation and migration compared with buprenorphine or placebo [11,12]. These previously described immunosuppressive effects have not been observed for hydromorphone, oxycodone and buprenorphine when used at equianalgesic doses [11,12]. Although the precise mechanism for this variability remains unclear, it has been hypothesized that the lack of immunosuppressive effects observed for some opioids is due to their different chemical structure compared with morphine [11,12].

Furthermore, the immunosuppressive effects of opioid analgesics have been shown to render exposed animals more susceptible to infections. Morphine-exposed mice were more likely to develop invasive Streptococcus pneumoniae infection compared with unexposed mice [11,12]. Similarly, several studies have demonstrated that opioid-exposed mice have an increased susceptibility to infection by Listeria monocytogenes, Salmonella enterica and Acinetobacter baumannii compared with unexposed mice. Some other animal model studies have further suggested that opioid exposure may induce greater susceptibility to infections caused by Gram-positive versus Gram-negative bacteria $[11,12]$.

\section{Limited data from early human studies on opioid use \& the risk of infections}

Although individuals using heroin (an illicit opioid with described immunosuppressive properties) have long been observed to have high infection rates, the presence of other contributing risk factors for infections made it difficult to elucidate the direct impact of the opioid exposure on the infection risk of those patients [14]. Regarding prescription opioid use, some small prior studies have reported an increased risk of infections among postsurgical, burn, cancer and intensive care patients using opioids [15-17]. However, the small sample sizes and the complexity of these specialized patient populations and settings limited the generalizability of those findings to broader populations.

\section{Supporting evidence from recent studies among humans}

More recently, three studies have evaluated the association between opioid analgesic use and the risk of serious infections in the general population. These studies have consistently reported a higher risk of infections associated with opioid analgesic use compared with no use. A case-control study conducted among older adults enrolled in a private health insurance system reported a 39\% increased odds of current opioid exposure among pneumonia cases compared with controls [18]. Similarly, in a study conducted among patients with rheumatoid arthritis enrolled in a State Medicaid program, periods of current opioid use were associated with a $38 \%$ increased risk of serious infections (including pneumonia, bacteremia, meningitis/encephalitis, septic arthritis/osteomyelitis, endocarditis, pyelonephritis and cellulitis) compared with periods of non-opioid use [19]. More recently, a nested case-control study, conducted in a population of subjects aged 5 years or older enrolled in a State Medicaid program, reported a $62 \%$ higher risk of laboratory-confirmed invasive pneumococcal disease among current users of opioids compared with past users [20]. Importantly, each of these studies consistently reported that patients using long-acting and high potency opioid formulations, opioids at high doses and opioids previously described as exhibiting immunosuppressive properties (i.e., morphine, codeine, fentanyl and methadone) had the highest risk for infection. The studies also noted a high risk for infections among patients recently initiating opioid use [18-20].

Opioid use was associated with an increased risk of multiple infection types across the three studies, demonstrating that the association cannot be fully explained by other opioid-related mechanisms which could contribute to infection (i.e., opioid-induced respiratory depression and microaspiration leading to pneumonia) [18-20]. Furthermore, each study applied strategies to account for potential protopathic bias (i.e., the possibility that individuals initiated opioid use to treat early symptoms of infection) and confounding by indication (i.e., individuals received opioids for an indication that is a risk factor for infection itself) [18-20]. In addition, in the study that examined the association between opioid use and the risk of laboratory-confirmed invasive pneumococcal disease, an extensive sensitivity analysis showed that residual confounding would be unlikely to explain the observed association [20]. The strength of the associations and the consistency of the findings in different populations and for different types of infection suggest that there is a clinically relevant association between opioid analgesic use and the risk of serious infections among humans, complementing the extensive evidence of opioid-induced immunosuppression from experimental animal studies.

\section{Future questions \& research challenges}

Although current evidence strongly suggests that opioid analgesic use is a risk factor for serious infections, additional evidence is needed to understand the observed associations better and to inform prescribing practices for opioid 
analgesics. Although the risk of infection is highest in the immediate period after opioid initiation, additional studies are needed to determine if the risk of infection associated with opioid use changes during periods of chronic exposure. Future studies are also needed to examine the risk of serious infection associated with individual opioids (immunosuppressive vs. non-immunosuppressive opioids and comparisons of individual opioids). Although opioid analgesics are already approved for use, new randomized controlled trials would be useful to characterize their full safety profile and to determine their appropriate role in pain management. Ideally, future experimental trials would encompass both acute and chronic opioid use, compare opioid use with appropriate control medications and be properly powered to study the risk of serious clinical outcomes, including infections. In the absence of new randomized experiments, additional epidemiological studies will be important to fill these knowledge gaps. Nevertheless, while additional evidence is generated, the existing evidence supports the judicious use of opioid analgesics in general, as well as an informed selection of specific types and formulations when treatment with opioid analgesics is warranted.

\section{Conclusion}

Opioid analgesic use is associated with an increased risk of serious infections and the risk varies by opioid type and dose. Providers and patients should consider the existing evidence and use appropriate caution when making prescribing decisions related to opioid analgesic use, especially for those already at high risk for serious infection.

\section{Financial \& competing interests disclosure}

This work was funded in part by the National Institutes of Health (R03 AG042981 and R01 AG043471). AD Wiese has no financial disclosures or conflicts of interest to report. CG Grijalva has received consulting fees from Pfizer and Merck and received research support from Sanofi-Pasteur, Campbell Alliance, the Centers for Disease Control and Prevention, National Institutes of Health, The Food and Drug Administration, and the Agency for Healthcare Research and Quality. The authors have no other relevant affiliations or financial involvement with any organization or entity with a financial interest in or financial conflict with the subject matter or materials discussed in the manuscript apart from those disclosed.

No writing assistance was utilized in the production of this manuscript.

\section{References}

1. Okie S. A flood of opioids, a rising tide of deaths. NEJM 363(21), 1981-1985 (2010).

2. Guy GP Jr, Zhang K, Bohm MK et al. Vital signs: changes in opioid prescribing in the United States, 2006-2015. MMWR Morb. Mortal. Wkly Rep. 66(26), 697-704 (2017).

3. Jani M, Dixon WG. Opioids are not just an American problem. BMJ (Clinical Res. Ed.) 359, j5514 (2017).

4. Dowell D, Haegerich TM, Chou R. CDC Guideline for prescribing opioids for chronic pain - United States, 2016. MMWR Recomm. Rep. 65(1), 1-49 (2016).

5. Benyamin R, Trescot AM, Datta S et al. Opioid complications and side effects. Pain Physician 11(Suppl. 2), S105-S120 (2008).

6. Dowell D, Haegerich TM, Chou R. CDC guideline for prescribing opioids for chronic pain - United States, 2016. JAMA 315(15), $1624-1645$ (2016).

7. Dart RC, Severtson SG, Bucher-Bartelson B. Trends in opioid analgesic abuse and mortality in the United States. NEJM 372(16), 1573-1574 (2015).

8. Ray WA, Chung CP, Murray KT, Hall K, Stein CM. Prescription of long-acting opioids and mortality in patients with chronic noncancer pain. JAMA 315(22), 2415-2423 (2016).

9. Ekstrom MP, Bornefalk-Hermansson A, Abernethy AP, Currow DC. Safety of benzodiazepines and opioids in very severe respiratory disease: national prospective study. BMJ (Clinical Res. Ed.) 348, g445 (2014).

10. Vozoris NT, Wang X, Fischer HD et al. Incident opioid drug use and adverse respiratory outcomes among older adults with COPD. Eur. Respir. J. 48(3), 683-693 (2016).

11. Plein LM, Rittner HL. Opioids and the immune system - friend or foe. Br. J. Pharmacol. doi:10.1111/bph.13750 (2017) (Epub ahead of print).

12. Sacerdote P. Opioid-induced immunosuppression. Curr. Opin. Support Palliat. Care 2(1), 14-18 (2008).

13. Sacerdote P, Manfredi B, Mantegazza P, Panerai AE. Antinociceptive and immunosuppressive effects of opiate drugs: a structure-related activity study. Br. J. Pharmacol. 121(4), 834-840 (1997).

14. Brack A, Rittner HL, Stein C. Immunosuppressive effects of opioids -clinical relevance. J. Neuroimmune Pharmacol. 6(4), 490-502 (2011). 
15. Shao YJ, Liu WS, Guan BQ et al. Contribution of opiate analgesics to the development of infections in advanced cancer patients. Clin. J. Pain 33(4), 295-299 (2017).

16. Inagi T, Suzuki M, Osumi M, Bito H. Remifentanil-based anaesthesia increases the incidence of postoperative surgical site infection. $J$. Hosp. Infect. 89(1), 61-68 (2015).

17. Schwacha MG, Mcgwin G Jr, Hutchinson CB, Cross JM, Maclennan PA, Rue LW 3rd. The contribution of opiate analgesics to the development of infectious complications in burn patients. Am. J. Surg. 192(1), 82-86 (2006).

18. Dublin S, Walker RL, Jackson ML et al. Use of opioids or benzodiazepines and risk of pneumonia in older adults: a population-based case-control study. J. Am. Geriatr. Soc. 59(10), 1899-1907 (2011).

19. Wiese AD, Griffin MR, Stein CM, Mitchel EF Jr, Grijalva CG. Opioid analgesics and the risk of serious infections among patients with rheumatoid arthritis: a self-controlled case series study. Arthritis Rheumatol. 68(2), 323-331 (2016).

20. Wiese AD, Griffin MR, Schaffner W et al. Opioid analgesic use and risk for invasive pneumococcal diseases: a nested case-control study. Ann. Intern. Med. doi:10.7326/m17-1907 (2018) (Epub ahead of print). 